\title{
Détermination des teneurs en polyphénols totaux, flavonoïdes totaux et tanins de jeunes feuilles non encore ouvertes de Piliostigma thonningii (Caesalpiniaceae)
}

\author{
Thomas Konan KOUAMÉ ${ }^{1}$, Sorho SIAKA ${ }^{1}$, Amian Brise Benjamin KASSI ${ }^{2}$ et \\ Yaya $\mathrm{SORO}^{1 *}$ \\ ${ }^{1}$ Laboratoire des Procédés Industriels de Synthèse, de l'Environnement et des Energies Nouvelles (LAPISEN), \\ Institut National Polytechnique Félix HOUPHOUËT-BOIGNY de Yamoussoukro, BP 1093 Yamoussoukro, \\ Côte d'Ivoire. \\ ${ }^{2}$ Laboratoire de Chimie Organique et Substances Naturelles (LCOSN), UFR-SSMT, Université Félix \\ Houphouët-Boigny, 22 BP 582 Abidjan 22, Côte d'Ivoire. \\ "Auteur correspondant ; E-mail : soro_y@yahoo.fr ; Tel : (00225) 07716766
}

Received: 05-11-2020 Accepted: 21-02-2021 $\quad$ Published: 28-02-2021

\section{RESUME}

Les jeunes feuilles non encore ouvertes de Piliostigma thonningii sont expressément utilisées par des tradipraticiens de Côte d'Ivoire pour la prise en charge de diverses pathologies. La présente étude visait à déterminer les teneurs en polyphénols totaux, en flavonoïdes et en tanins condensés d'extraits et de fractions de ces jeunes feuilles non encore ouvertes. Leurs métabolites secondaires ont été extraits par macération dans un mélange éthanol-eau (70/30: v/v) avec un rendement de 36\%. Le fractionnement successif de l'extrait hydroalcoolique obtenu par l'hexane, le dichlorométhane, l'acétate d'éthyle, l'éthanol et l'eau a donné des rendements variant de 5,55 à 33,33\%. Le dosage des polyphénols totaux, des flavonoïdes et des tanins condensés a donné des teneurs variant entre $0,23 \pm 0,05$ et 146,67 $\pm 4,25 \mathrm{mg}$ EAG/g d'extrait sec, entre 5,33 $\pm 0,62$ et 112,11 $\pm 0,83 \mathrm{mg} \mathrm{EQ} / \mathrm{g}$ d'extrait sec et entre $23,33 \pm 0,94$ et $38,33 \pm 0,47 \mathrm{mg}$ EAT/g d'extrait sec respectivement. De façon générale, l'extrait hydroalcoolique et la fraction à l'acétate d'éthyle sont les plus riches en métabolites secondaires, en polyphénols, en flavonoïdes et en tanins condensés.

() 2021 International Formulae Group. All rights reserved.

Mots clés : Piliostigma thonningii, rendements, polyphénols totaux, flavonoïdes, tanins.

\section{Determination of the contents of total polyphenols, total flavonoids and tannins in young, unopened leaves of Piliostigma thonningii (Caesalpiniaceae)}

\begin{abstract}
The young, unopened leaves of Piliostigma thonningii are specifically used by traditional practitioners in Côte d'Ivoire for the treatment of various pathologies. The present study aimed to determine the contents of total polyphenols, flavonoids and condensed tannins of extracts and fractions of these young, unopened leaves.
\end{abstract}


Their secondary metabolites were extracted by maceration in an ethanol-water mixture (70/30: v/v) with a yield of $36 \%$. The successive fractionation of the hydroalcoholic extract obtained by hexane, dichloromethane, ethyl acetate, ethanol and water gave yields varying from 5.55 to $33.33 \%$. The determination of total polyphenols, total flavonoids and condensed tannins gave contents varying between $0.23 \pm 0.05$ and $146.67 \pm 4.25 \mathrm{mg}$ EAG / g of dry extract, between $5.33 \pm 0.62$ and $112.11 \pm 0.83 \mathrm{mg}$ EQ / $\mathrm{g}$ of dry extract and between $23.33 \pm 0.94$ et 38.33 $\pm 0.47 \mathrm{mg} \mathrm{EAT} / \mathrm{g}$ of dry extract respectively. In general, the hydroalcoholic extract and the ethyl acetate fraction are the richest in secondary metabolites, polyphenols, flavonoids and condensed tannins.

(C) 2021 International Formulae Group. All rights reserved.

Keywords: Piliostigma thonningii, yields, total polyphenols, flavonoids, tannins.

\section{INTRODUCTION}

Les populations du monde et particulièrement celles de l'Afrique sont de plus en plus confrontées à la résurgence de certaines maladies telles que les maladies cardiovasculaires, le cancer et le diabète (Aseervatham et al., 2013). Face à cette situation, de nombreux travaux sont réalisés en vue de trouver de nouvelles sources de biomolécules dans les plantes médicinales (Soro et al., 2012 ; Liu et al., 2017). En effet, ces plantes servent de remèdes contre les maladies (Goly et al., 2015 ; Coulibaly et al., 2019) et sont utilisées en thérapie par environ $80 \%$ de la population des pays en développement (OMS, 2003). Ainsi, les propriétés biologiques des extraits de plantes ont fait l'objet de plusieurs travaux scientifiques (Golly et al., 2015 ; Coulibaly et al., 2020).

Piliostigma thonningii est une plante de la famille des Caesalpiniaceae utilisée dans la pharmacopée pour ses nombreuses propriétés biologiques. Ses feuilles fraîches sont utilisées dans le traitement des ulcères (Ukwuani et al., 2012) et de la malaria (Madara et al., 2010). Elles possèdent des propriétés antiinflammatoires, antibactériennes (Pousset, 2006), antifongiques (Agban et al., 2013) et antioxydantes (Dieng et al., 2017). Les jeunes feuilles non encore ouvertes de Piliostigma thonningii, contrairement aux jeunes feuilles déjà ouvertes, sont expressément utilisées par des tradipraticiens de Côte d'Ivoire pour la prise en charge de diverses pathologies. Les propriétés biologiques des plantes sont généralement attribuées à leurs teneurs en composés phénoliques, particulièrement aux flavonoïdes et aux tanins (Santos-Sanchez et al., 2014 ; Pavun et al., 2018). La détermination de ces teneurs dans les extraits de plantes apparaît donc comme un indicateur de leurs activités biologiques.

La présente étude visait à déterminer les teneurs en polyphénols totaux, en flavonoïdes et en tanins condensés d'extraits et de fractions de jeunes feuilles non encore ouvertes de Piliostigma thonningii acclimaté en Côte d'Ivoire. Il n'existe, à notre connaissance, aucune étude réalisée sur les jeunes feuilles non ouvertes de cette plante.

\section{MATERIEL ET METHODES Matériel végétal}

Les jeunes feuilles non encore ouvertes de Piliostigma thonningii ont été récoltées avant le lever du soleil en mai 2018 à Bouaké (6047'18.762” Nord et 5015'25.9992”' Ouest) au centre de la Côte d'Ivoire et identifiées par monsieur Amani N'Guessan, botaniste à l'Institut National Polytechnique Félix HOUPHOUËT-BOIGNY (INP-HB) de Yamoussoukro. Les feuilles ont été séchées à l'ombre à la température ambiante du laboratoire $\left(26\right.$ à $30^{\circ} \mathrm{C}$ ) pendant 14 jours puis broyées. Les poudres obtenues ont été tamisées à l'aide d'un tamis de $0,5 \mathrm{~mm}$ de maille et conservées dans des bocaux colorés à $4{ }^{\circ} \mathrm{C}$, à l'abri de la lumière et de l'humidité jusqu'à leur utilisation ultérieure. 


\section{Procédure d'extraction et de fractionnement du matériel végétal}

Le matériel végétal a été extrait dans un mélange éthanol/eau (70/30) selon la méthode décrite par Kassi et al. (2014). Après extraction, une masse de $25 \mathrm{~g}$ de l'extrait hydroalcoolique a été dissoute dans $250 \mathrm{~mL}$ d'eau à $60{ }^{\circ} \mathrm{C}$ et fractionnée successivement avec $2 \times 250 \mathrm{~mL}$ d'hexane, de dichlorométhane et d'acétate d'éthyle. La phase aqueuse résultante a été évaporée pour éliminer l'eau et séchée à l'étuve à $50^{\circ} \mathrm{C}$ pendant $4 \mathrm{~h}$. Le résidu résultant a été repris avec 2 x $250 \mathrm{~mL}$ d'éthanol. Le résidu non soluble dans l'alcool, qui constitue la fraction aqueuse, a été séché à l'étuve à $50{ }^{\circ} \mathrm{C}$. Les différentes phases organiques obtenues ont été séparément séchées sur sulfate de sodium anhydre. Après filtration et élimination des solvants sous pression réduite, les fractions à l'hexane (FHE), au dichlorométhane (FDM), à l'acétate d'éthyle (FAE), à l'éthanol (FET) obtenues ont été conservées pour une utilisation ultérieure. Le rendement $(\mathrm{R})$ de l'extrait est déterminé par le ratio de la masse de l'extrait hydroalcoolique sec (m) par la masse de poudre utilisée (MT) : $\mathrm{R}(\%)=\mathrm{m} \times 100 / \mathrm{MT}$. Les rendements des fractions sont déterminés selon la même formule.

\section{Dosages des polyphénols totaux}

Le dosage des polyphénols totaux a été réalisé selon la méthode décrite par Wood et al. (2002). A un volume de $30 \mu \mathrm{L}$ d'extrait ou de fraction ont été ajoutés $2,5 \mathrm{~mL}$ de réactif de Folin-Ciocalteu dilué au $1 / 10^{\mathrm{e}}$. Le mélange obtenu a été maintenu pendant $2 \min$ à l'obscurité à la température ambiante $(27 \pm 3$ $\left.{ }^{\circ} \mathrm{C}\right)$ puis $2 \mathrm{~mL}$ de solution de carbonate de sodium à $75 \mathrm{~g} / \mathrm{L}$ y ont été ajoutés. La solution obtenue a été ensuite incubée à $50{ }^{\circ} \mathrm{C}$ pendant 15 min. La lecture de l'absorbance a été réalisée au spectrophotomètre UV-visible à une longueur d'onde de $760 \mathrm{~nm}$ contre un blanc constitué de $5 \mathrm{~mL}$ de réactif de Folin-Ciocalteu dilué au $1 / 10^{\mathrm{e}}$ et de $4 \mathrm{~mL}$ de la solution de carbonate de sodium à $75 \mathrm{~g} / \mathrm{L}$. L'acide gallique a été utilisé comme standard de référence pour l'établissement de la courbe d'étalonnage et pour la quantification des teneurs en polyphénols totaux exprimées en $\mathrm{mg}$ d'équivalent d'acide gallique par gramme d'extrait (mg EAG/g d'extrait). Les essais ont été réalisés en triplé pour chaque échantillon.

Les teneurs en polyphénols totaux de l'extrait hydroalcoolique et des fractions ont été déterminées à partir de la courbe d'étalonnage $\mathrm{Y}=1,129 \mathrm{X}+0,0544$ avec $\mathrm{R}^{2}=$ 0,9967 tracée en utilisant l'acide gallique comme standard.

\section{Dosages des flavonoïdes totaux}

La teneur en flavonoïdes totaux a été déterminée selon la méthode décrite par Marinova et al. (2005). Des volumes de 0,75 $\mathrm{mL}$ de solution de nitrite de sodium à $5 \%(\mathrm{~m} / \mathrm{v})$ et de $0,75 \mathrm{~mL}$ de solution de chlorure d'aluminium à $10 \%(\mathrm{~m} / \mathrm{v})$ ont été ajoutés à 2,5 $\mathrm{mL}$ de solution d'extrait ou de fraction à $1 / 500$ $(\mathrm{m} / \mathrm{V})$. Après $5 \mathrm{~min}$ d'incubation, le mélange a été mis en contact avec $5 \mathrm{~mL}$ d'une solution de soude à $1 \mathrm{M}$. Le volume obtenu a été ajusté à $25 \mathrm{~mL}$ puis agité vigoureusement. L'absorbance a été mesurée à une longueur d'onde de $510 \mathrm{~nm}$ contre un blanc constitué de $0,75 \mathrm{~mL}$ de solution de nitrate de sodium à $5 \%$ $(\mathrm{m} / \mathrm{v})$, de $0,75 \mathrm{~mL}$ de solution de chlorure d'aluminium à $10 \%(\mathrm{~m} / \mathrm{v})$ et de $5 \mathrm{~mL}$ de solution de soude à $1 \mathrm{M}$. La quercétine a été utilisée comme standard de référence pour l'établissement de la courbe d'étalonnage et pour la quantification des teneurs en flavonoïdes totaux exprimées en milligramme d'équivalent de quercétine par gramme d'extrait (mg EQ/g d'extrait). Les essais ont été réalisés en triplé pour chaque échantillon.

Les teneurs en flavonoïdes de l'extrait hydroalcoolique et des fractions ont été déterminées à partir de la courbe d'étalonnage $\mathrm{Y}=0,6455 \mathrm{X}-0,0079$ avec $\mathrm{R}^{2}=0,9983$ tracée en utilisant la quercétine comme standard. 


\section{Dosage des tanins condensés}

La teneur en tanins condensés a été déterminée selon la méthode décrite par Julkunen-Titto (1985). Une quantité de $50 \mathrm{~mL}$ de chaque fraction ou de l'extrait hydroalcoolique a été ajoutée à $1500 \mu \mathrm{L}$ de la solution à $4 \%$ de vanilline dans le méthanol. Le mélange résultant est vigoureusement agité et un volume de $750 \mu \mathrm{L}$ d'acide chlorhydrique concentré y a été additionné. Le mélange obtenu est laissé au repos pour réagir à température ambiante pendant $20 \mathrm{~min}$. L'absorbance a été mesurée à une longueur d'onde de $550 \mathrm{~nm}$ contre un blanc constitué de la solution à $4 \%$ de vanilline dans le méthanol. Les essais ont été réalisés en triplé pour chaque échantillon. Une solution mère d'acide tanique a été utilisée comme standard de référence pour l'établissement de la courbe d'étalonnage et pour la quantification des teneurs en tanins condensés exprimés en milligramme équivalent d'acide tanique par gramme de la matière sèche (mg EAT/g de la matière sèche). Les essais ont été réalisés en triplé pour chaque échantillon.

Les teneurs en tanins condensés de l'extrait hydroalcoolique et des fractions ont été déterminées à partir de la courbe d'étalonnage $\mathrm{Y}=0,1165 \mathrm{X}+0,00003$ avec $\mathrm{R}^{2}$ $=0,9996$ tracée en utilisant la solution mère d'acide tanique comme standard.

\section{RESULTATS}

\section{Rendements de l'extraction et des fractionnements}

Les rendements de l'extrait hydroalcoolique et des différentes fractions sont présentés dans la Figure 1. Il ressort de cette figure que le rendement de l'extrait hydroalcoolique (EHA) des jeunes feuilles non encore ouvertes de Piliostigma thonningii est de $36 \%$. Par ailleurs, les rendements des fractions varient de $5,55 \%$ pour la fraction éthanolique (FET) à 33,33\% pour la fraction à l'acétate d'éthyle (FAE). Les meilleurs rendements sont obtenus pour les fractions à l'acétate d'éthyle (FAE) et au dichlorométhane (FDM) avec des valeurs de 33,33 et $27,77 \%$ respectivement.

\section{Teneurs en polyphénols totaux}

Les résultats obtenus sont présentés dans la Figure 2. Les teneurs en polyphénols totaux varient entre $0,23 \pm 0,04$ et $146,67 \pm$ 4,24 mg EAG/g d'extrait sec respectivement pour la fraction aqueuse et l'extrait hydroalcoolique. Les teneurs en polyphénols des fractions éthanolique, à l'hexane et au dichlorométhane sont faibles avec des valeurs respectives de $1,83 \pm 0,12 ; 1,67 \pm 0,04$ et 1,17 $\pm 0,04 \mathrm{mg}$ EAG/g d'extrait sec.

\section{Teneurs en flavonoïdes totaux}

Les résultats obtenus sont présentés dans la Figure 3. Les teneurs en flavonoïdes varient entre $5,33 \pm 0,62$ et $112,11 \pm 0,83 \mathrm{mg}$ $\mathrm{EQ} / \mathrm{g}$ d'extrait sec respectivement pour les fractions au dichlorométhane et à l'acétate d'éthyle. La teneur de la fraction à l'acétate d'éthyle est plus élevée que celle de l'extrait hydroalcoolique $(68,33 \pm 0,94 \mathrm{mg} \mathrm{EQ} / \mathrm{mg}$ d'extrait sec) alors que les fractions éthanolique et aqueuse ont des teneurs respectives de $22,00 \pm 1,08$ et $16,67 \pm 0,94 \mathrm{mg}$ $\mathrm{EQ} / \mathrm{g}$ d'extrait sec.

\section{Teneurs en tanins condensés}

Les résultats obtenus sont présentés dans la Figure 4. Les teneurs en tanins condensés varient entre 23,33 $\pm 0,94$ et 38,33 \pm $0,47 \mathrm{mg}$ EAT/g d'extrait sec respectivement pour la fraction éthanolique et l'extrait hydroalcoolique. La teneur la plus élevée au niveau des fractions est celle de la fraction à l'acétate d'éthyle avec 36,67 $\pm 0,47 \mathrm{mg}$ EAT/g d'extrait sec, suivie des fractions au dichlorométhane et à l'hexane qui ont respectivement des teneurs de 27,67 $\pm 1,24$ et $24,67 \pm 1,24 \mathrm{mg}$ EAT/g d'extrait sec. 


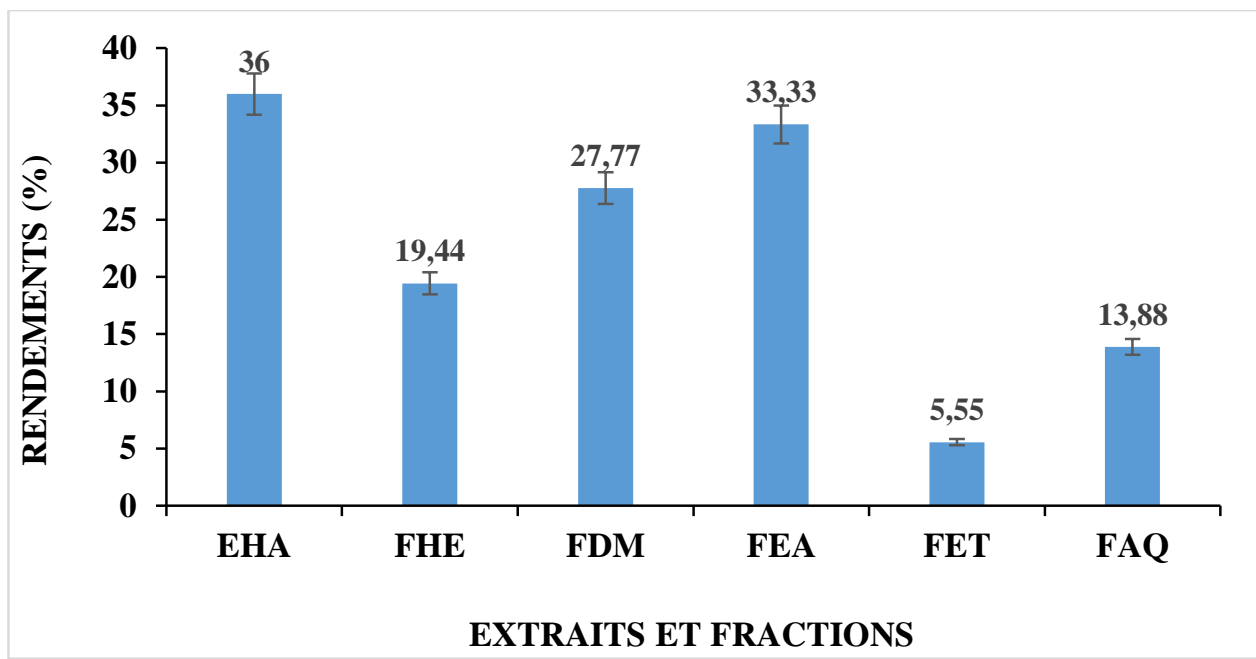

Figure 1 : Rendements de l'extrait hydroalcoolique et des fractions des jeunes feuilles non encore ouvertes de Piliostigma thonningii de Côte d'Ivoire.

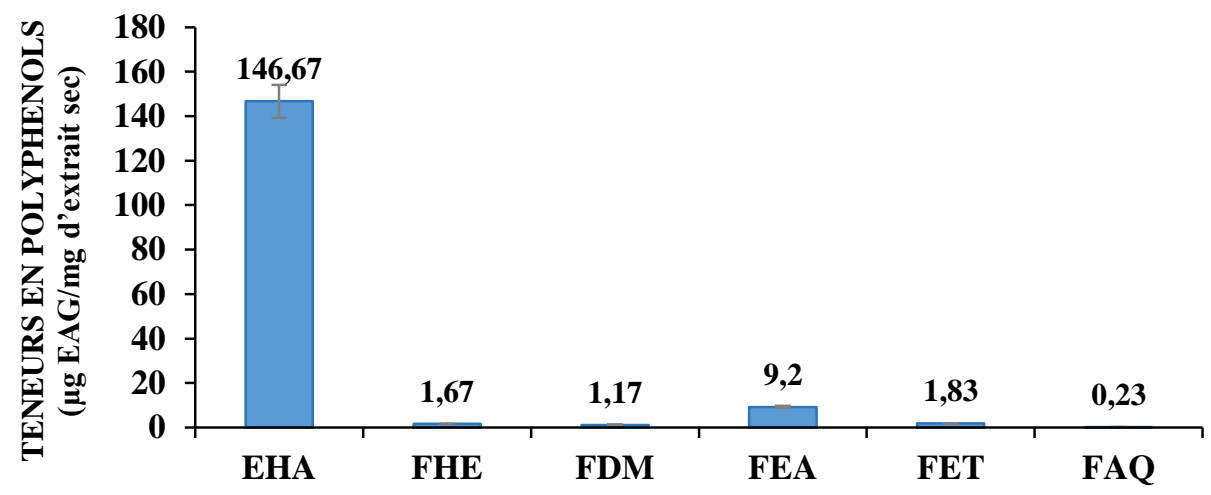

EXTRAITS ET FRACTIONS

Figure 2 : Teneurs en polyphénols totaux de l'extrait hydroalcoolique et des fractions des jeunes feuilles non encore ouvertes de Piliostigma thonningii de Côte d'Ivoire.

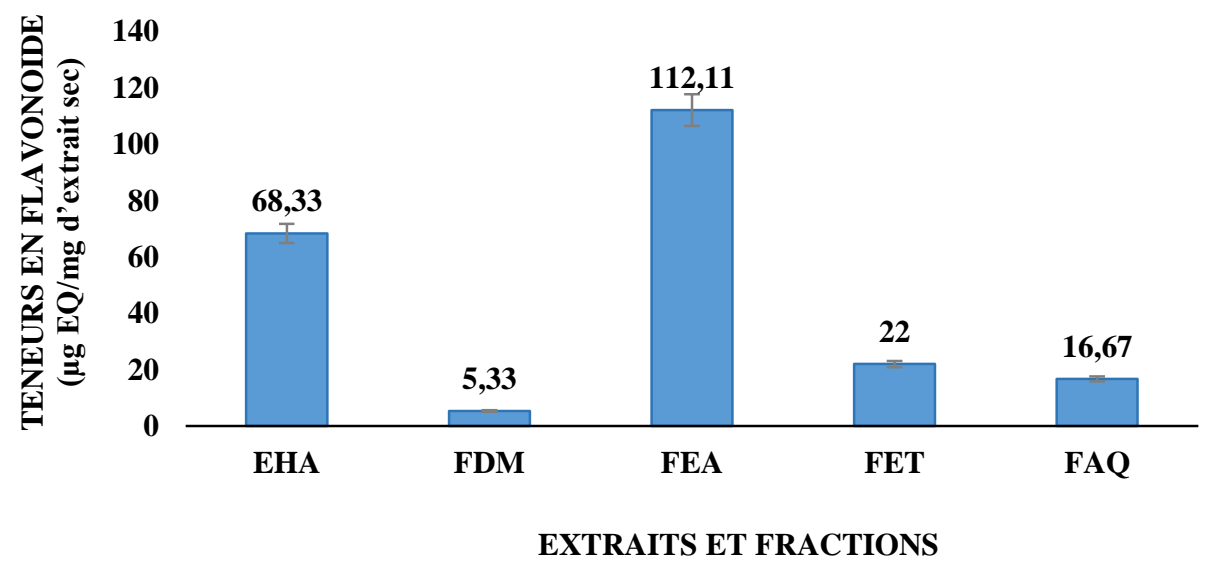


Figure 3 : Teneurs en flavonoïdes de l'extrait hydroalcoolique et des fractions des jeunes feuilles non encore ouvertes de Piliostigma thonningii de Côte d'Ivoire.

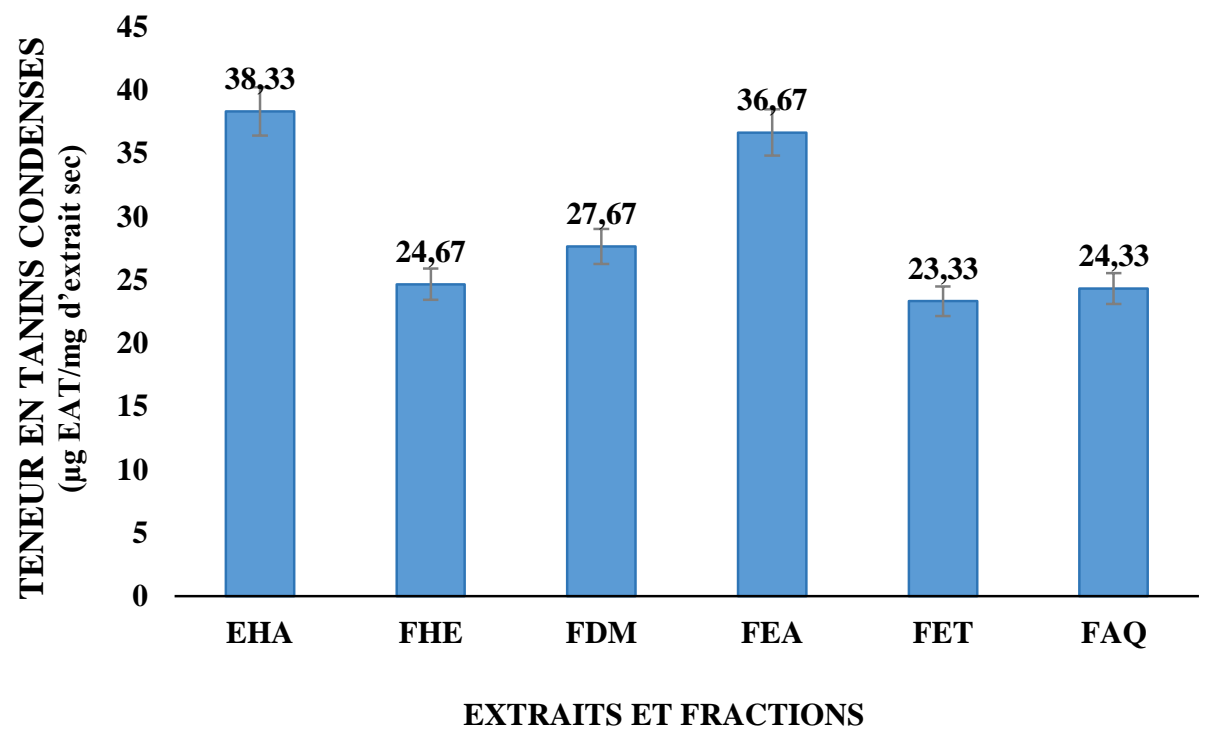

Figure 4 : Teneurs en tanins condensés de l'extrait hydroalcoolique et des fractions des jeunes feuilles non encore ouvertes de Piliostigma thonningii de Côte d'Ivoire.

\section{DISCUSSION}

Les meilleurs rendements, obtenus pour les fractions à l'acétate d'éthyle (FAE) et au dichlorométhane (FDM) avec des valeurs de 33,33 et $27,77 \%$ respectivement, suggèrent une plus grande présence de métabolites secondaires moyennement polaires dans l'extrait hydroalcoolique des jeunes feuilles non ouvertes de Piliostigma thonningii. Les fractions éthanolique (FET) et aqueuse (FAQ) ont les plus faibles rendements certainement dû à l'épuisement de l'extrait hydroalcoolique par les solvants précédents. Nos rendements des fractions au dichlorométhane, à l'éthanol et à l'eau sont supérieurs à ceux obtenus par Agban et al. (2013) et qui sont de 0,$30 ; 2,80$ et $2,40 \%$ respectivement pour les extraits au dichlorométhane, à l'éthanol et à l'eau des feuilles de cette plante acclimatée au Togo.

Nos rendements des fractions à l'hexane, à l'acétate d'éthyle et à l'eau sont également supérieurs à ceux obtenus par Ukwuani et al. (2012) et qui sont respectivement de 4,$5 ; 7,2 ; 8,4 \%$ pour la plante acclimatée au Nigéria. Toutefois, nos rendements en extraits aqueux et éthanolique sont inférieurs à ceux de Ahomadegbe et al. (2018) et Ibrahim et al. (2019) qui sont respectivement de 17,11/15,5\% et 19,29 / $31,3 \%$ pour les plantes acclimatées au Bénin/Nigéria. Cette variabilité des rendements pourrait être due à la méthode d'extraction (directe ou successive), à la nature de la matière végétale (feuilles mature ou jeune, ouvertes ou non) ou à des facteurs intrinsèques tels que le climat et la nature du sol. En effet, les études réalisées par Ibrahim et al. (2019) sur les jeunes feuilles et les feuilles matures de $P$. thonningii acclimatée au Nigéria ont montré que les rendements des extraits éthanolique et aqueux obtenus avec les jeunes feuilles étaient plus élevés que ceux obtenus avec ces mêmes extraits pour les feuilles matures.

La teneur en polyphénols totaux de notre extrait hydroalcoolique issus des jeunes feuilles non ouvertes de Piliostigma thonningii $(146,67 \pm 4,24 \mathrm{mg}$ EAG/g d'extrait sec) est supérieure à celle de Dieng et al. (2017) qui ont obtenu 3,12 $\pm 0,08 \mathrm{mg}$ EAT/g d'extrait sec pour les feuilles matures de $P$. thonningii acclimaté au Sénégal. Ces résultats pourraient 
suggérer la plus grande richesse des jeunes feuilles non encore ouvertes en polyphénols totaux. Au niveau des fractions, la teneur la plus élevée est obtenue avec l'acétate d'éthyle $(9,20 \pm 0,32 \mathrm{mg} E A G / g$ d'extrait sec) suivie des fractions éthanolique (obtenue après une extraction avec des solvants de polarité croissante) et hexanique qui ont respectivement des valeurs de $1,83 \pm 0,12$ et $1,67 \pm 0,04 \mathrm{mg}$ EAG/g d'extrait sec. Le fractionnement a donc permis une répartition des polyphénols totaux dans toutes les fractions en concentrant le plus grand nombre dans la fraction à l'acétate d'éthyle, moyennement polaire. Ces polyphénols pourraient être donc majoritairement sous une forme de génines libres (Muyonga et al., 2014).

La teneur en flavonoïdes de l'extrait hydroalcoolique des jeunes feuilles non encore ouvertes de Piliostigma thonningii $(68,33 \pm$ $0,94 \mathrm{mg} \mathrm{EQ} / \mathrm{g}$ d'extrait sec) est 34 fois supérieure à la teneur $(2,17 \pm 0,13 \mathrm{mg} \mathrm{EQ} / \mathrm{g}$ d'extrait sec) dans les feuilles matures de $P$. thonningii obtenue par Sombie et al. (2018) au Burkina Faso. Les résultats des teneurs en flavonoïdes dans les fractions montrent qu'elles dépendent de la maturité des feuilles et de leurs stades d'évolution (feuilles ouvertes ou non), mais également de la polarité des solvants utilisés comme rapportés par Hami et al. (2014). Ces flavonoïdes pourraient être donc majoritairement sous une forme de génines libres (Muyonga et al., 2014).

La teneur en tanins condensés de l'extrait hydroalcoolique des jeunes feuilles non encore ouvertes de Piliostigma thonningii $(38,33 \pm 0,47 \mathrm{mg}$ EAT/g d'extrait sec) est largement supérieure à la teneur $(12,62 \pm 0,02$ $\mathrm{mg} \mathrm{EAT/g} \mathrm{d'extrait} \mathrm{sec)} \mathrm{obtenue} \mathrm{par} \mathrm{Sombie} \mathrm{et}$ al. (2018) dans les feuilles matures de $P$. thonningii acclimaté du Burkina Faso. Ces résultats montrent que les tanins sont concentrés dans les jeunes feuilles non encore ouvertes. La teneur en tanins des fractions est plus élevée dans les solvants non polaires laissant suggérer des tanins libres.

\section{Conclusion}

L'étude réalisée sur les jeunes feuilles non encore ouvertes de Piliostigma thonningii a permis de mettre en évidence la présence de plusieurs familles de métabolites secondaires. La fraction à l'acétate d'éthyle est la plus riche des fractions avec un rendement en métabolites secondaires de 33,33\%. Les polyphénols totaux, les flavonoïdes et les tanins condensés sont plus abondants dans la fraction à l'acétate d'éthyle issue de l'extrait hydroalcoolique. Cette étude montre que les jeunes feuilles non encore ouvertes sont plus intéressantes que les feuilles matures ou les jeunes feuilles ouvertes.

\section{CONFLIT D'INTERETS}

Les auteurs déclarent qu'il n'existe aucun conflit d'intérêts entre eux ou autre partie au sujet de cet article.

\section{CONTRIBUTIONS DES AUTEURS}

Nous déclarons que ce travail a été réalisé par les auteurs cités dans cet article et toutes les responsabilités relatives aux réclamations concernant le contenu de cet article seront à la charge des auteurs. La récolte des feuilles de la plante utilisée, l'extraction et le fractionnement du matériel végétal ainsi que les différents dosages ont été effectués par TKK et ABBK. La supervision des travaux et la rédaction de l'article ont été faites par SS et YS.

\section{REFERENCES}

Agban A, Gbogbo KA, Hoekou YP, Atchou K, Tchacondo T, Batawila K, De Souza C, Gbeassor M. 2013. Évaluation de l'activité antifongique des extraits de Cassia alata L. et de Piliostigma thonningii (Schumach.) Milne Redh. (Fabaceae) sur Candida albicans. Int. J. Biol. Chem. Sci., 7(3): 1041-1047. DOI: http://dx.doi.org/10.4314/ijbcs.v7i3.12

Ahomadegbe MA, Ladekan EY, Togbenou N, Assogba F, Agbonon A, Gbenou J. 2018. Phytochemical and toxicity studies of the leaves of Mangifera indica, Cajanus Cajan and of Piliostigma thonningii, acclimated in Benin, used against diarrheal disease. Journal of Pharmacognosy and Phytochemistry, 7(2): 2971-2978. 
Aseervatham GS, Sivasudha T, Jeyadevi R, Arul AD. 2013. Environmental factors and unhealthy lifestyle influence oxidative stress in humans-an overview. Environ Sci Pollut Res., 20(7): 43564369.

DOI: http://dx.doi.org/10.1007/s11356-0131748-0

Coulibaly O, Ira B, Soro Y, Dosso M, Touré A, Yapo-Crezoit C. 2019. Activités antifongiques des fractions de l'extrait hydroalcoolique de Hugonia platysepala sur les germes responsables de cryptococcoses opportunistes du VIH/SIDA. Phytothérapie, 1-7. DOI: http://dx.doi.org/10.3166/phyto-20190218

Coulibaly O, Yapo-crezoit C, Ira B, Toure A, Soro Y. 2020. Evaluation de l'activité antifongique des extraits totaux de Hugonia platysepala sur les pathogènes responsables de cryptococcoses chez les sujets infectés par le VIH. J. Appl. Biosci., 146: 15046-15054. DOI: https://doi.org/10.35759/JABs.v146.7

Dieng SIM, Fall AD, Diatta-Badji K, Sarr A, Sene M, Sene M, Mbaye A, Diatta W, Bassene E. 2017. Evaluation de l'activité antioxydante des extraits hydroéthanoliques des feuilles et écorces de Piliostigma thonningii Schumach. Int. J. Biol. Chem. Sci., 11(2): 768-776. DOI: https://dx.doi.org/10.4314/ijbcs.v11i2.19

Golly KJ, Siaka S, Soro Y, Guessennd N, Dosso M, Djaman AJ. 2015. Phytochemical Study and Antimicrobial Activity of Bark Extracts of Ceiba pentandra (L.) Gaertn. (Bombacaceae) from Côte d'Ivoire on Antibiotic Resistant Staphylococcus aureus and Pseudomonas aeruginosa. British Microbiology Research Journal, 9(1): 17.

Goly C, Soro Y, Kassi B, Dadié A, Soro S, Djé M. 2015. Antifungal activities of the essential oil extracted from the tea of savanna (Lippia multiflora) in Côte d'Ivoire. Int. J. Biol. Chem. Sci., 9(1): 2434.

DOI: http://dx.doi.org/10.4314/ijbcs.v9i1.3
Hamia C, Guergab A, Rennane NE, Birache M, Haddad M, Saidi M, Yousfi M. 2014. Influence des solvants sur le contenu en composés phénoliques et l'activité antioxydante des extraits du Rhanterium adpressium. Annales des Sciences et Technologie, 6(1) : 33-39.

Ibrahim IL, Musah M, Dagaci MZ, Mohammed SH, Baba HF, Umar MT, Usman RL. 2019. Phytochemical screening, mineral determination and antimicrobial screening of the leaves extracts of Piliostigma thonningii (matured and young) leaves. African Journal of Agriculture and Food Science, 2(1): 15-27.

Julkunen-Titto R. 1985. Phenolic constituents in the leaves of Northern Willows: Methods for the Analysis of Certain Phenolics. J. Agric. Food Chem., 33: 213217.

DOI: https://doi.org/10.1021/jf00062a013

Kassi ABB, Soro Y, Fanté B, Golly KJ, Sorho S, Touré AS, Coustard J-M. 2014. Isolation and identification of bioactive compounds from kernel seed cake of the mango (Mangifera indica Lam). Int. J. Biol. Chem. Sci., 8(4): 1885-1895. DOI: http://dx.doi.org/10.4314/ijbcs.v8i4.48

Liu Z, Mo K, Fei S, Zu Y, Yang L. 2017. Efficient approach for the extraction of proanthocyanidins from Cinnamomum longepaniculatum leaves using ultrasonic irradiation and an evaluation of their inhibition activity on digestive enzymes and antioxidant activity in vitro. Journal of Separation Science, 40(15): 31003113.

DOI: http://dx.doi.org/10.1002/jssc.201700342

Madara AA, Ajayi JA, Salawu OA, Tijani AY. 2010. Anti-malarial activity of ethanolic leaf extract of Piliostigma thonningii Schum. (Caesalpiniacea) in mice infected with Plasmodium berghei berghei. African Journal of Biotechnology, 9(23): 3475-3480.

DOI: http://dx.doi.org/10.4314/njpar.v31i2.69 476

Marinova D, Ribarova F, Atanassova M. 2005. Total phenolics and total flavonoids in 
bulgarian fruits and vegetables. Journal of the University of Chemical Technology and Metallurgy, 40(3): 255-260.

Muyonga JH, Andabati B, Ssepuuya G. 2014. Effect of heat processing on selected grain amaranth physicochemical properties. Food Science \& Nutrition, 2(1): $9-16 . \quad$ DOI: http://dx.doi.org/10.1002/fsn3.75.

OMS. 2003. Médicaments essentiels et politiques pharmaceutiques : donner un soutien aux pays pour réduire le manque d'accès aux médicaments. Rapport annuel 2002, Genève, 20 p.

Ouattara A, Traore Y, Ouattara GA, Konaté G, Ouattara K, Coulibaly A. 2020. Antioxidant and anti-gastroenteritis activities of Funtumia elastica (Apocynaceae) and Caesalpinia bonduc (Caeasalpiniaceae). Int. J. Biol. Chem. Sci., 14(1): 170-180. DOI: https://dx.doi.org/10.4314/ijbcs.v14i1.14

Pavun L, Uskoković-Marković S, JelikićStankov M, Đikanović D, Đurđević P. 2018. Determination of Flavonoids and Total Polyphenol Contents in Commercial Apple Juices. Czech J. Food Sci., 36(3): 233-238. DOI: https://doi.org/10.17221/211/2017-CJFS

Pousset JL. 2006. Politiques nationales : place des médicaments traditionnels en Afrique. Méd. Trop., 66: 606-609.

Santos-Sanchez NF, Flores-Parra A, ValadezBlanco R, Fernandez-Rojas B, Martinez-
Vasquez JB, Salas-Coronado R. 2014. Polyphenolic content, free radical scavenging activity and isolation of Tiliroside from Heliocarpus terebinthinaceus (Tiliaceae) Seeds. Journal of Biological Sciences, 14(5): 376-380.

DOI: http://dx.doi.org/10.3923/jbs.2014.376.3 80

Sombie EN, Tibiri A, N'do JY-P, Traoré TK, Ouedroaogo N, Hilou A, Guissou PI, Nacoulma OG. 2018. Ethnobotanical study and antioxidant activity of antihepatitis plants extracts of the COMOE province, Burkina Faso. Int. J. Biol. Chem. Sci., 12(3): 1308-1319. DOI: https://dx.doi.org/10.4314/ijbcs.v12i3.19

Soro Y, Kassi ABB, Bamba F, Siaka S, Touré SA, Coustard J-M. 2012. Flavonoids and gallic acid from leaves of Santaloides afzelii (Connaraceae). Rasayan Journal of Chemistry, 5(3): 332-337.

Ukwuani AN, Ihebunna O, Samuel RM and Peni IJ. 2012. Acute Oral Toxicity and Antiulcer Activity of Piliostigma thonningii Leaf Fraction in Albino rats. Bull. Env. Pharmacol. Life Sci., 2(1): 4145.

Wood JE, Senthilmohana ST, Peskinb AV. 2002. Antioxidant activity of procyanidin-containing plant extracts at different pHs. Food Chemistry, 77(2): 155-161. 